\title{
Fractal quintic spline method for nonlinear boundary-value problems
}

\author{
N. Balasubramani*1 (D), M. Guru Prem Prasad ${ }^{2}$ (D), S. Natesan² (D) \\ ${ }^{1}$ Department of Mathematics, National Institute of Technology Puducherry, Karaikal 609609, India \\ ${ }^{2}$ Department of Mathematics, Indian Institute of Technology Guwahati, Assam 781039, India
}

\begin{abstract}
In this article, numerical solutions of nonlinear boundary-value problems are obtained using fractal quintic spline. Convergence analysis of the proposed method is also established. Proposed method has fourth-order convergence. Numerical examples are provided to show practical usefulness of the method and numerical results are compared with the existing numerical methods.
\end{abstract}

Mathematics Subject Classification (2010). 28A80, 65D07, 34B15

Keywords. fractal quintic spline, nonlinear boundary-value problem, quasilinearization, error estimate

\section{Introduction}

In this article, we consider the nonlinear boundary-value problems (BVPs) of the form

$$
\left\{\begin{array}{l}
u_{x x}(x)+F(x, u(x))=0, \quad x \in(0,1), \\
u(0)=\eta_{0}, \quad u(1)=\eta_{1}
\end{array}\right.
$$

where $\eta_{0}$ and $\eta_{1}$ are constants. We assume that for $(x, u(x)) \in D=\{0 \leq x \leq 1,-\infty<$ $u(x)<\infty\}$, the functions $F$ and $\partial F / \partial u$ are continuous. It is known that problem (1.1) admits a unique solution, provided $\sup \partial F / \partial u<\pi^{2}[20]$. Here, we assume that $\frac{\partial F}{\partial u} \leq 0$ on $D$ and $\frac{\partial F}{\partial u}<0$ on $D^{\circ}=\{0<x<1,-\infty<u(x)<\infty\}$. The notation $u_{x x}$ is used instead of $u^{\prime \prime}$ mainly for the sake of convenience in the future.

To obtain the numerical approximate solutions of nonlinear BVPs, various authors have used different methods. For example, Chawla [14] developed a fourth-order numerical method using cubic spline to get the numerical solutions of nonlinear BVPs. Ravikanth [23] used the cubic spline and Rashidinia [22] used non-polynomial cubic spline to obtain the numerical solutions of nonlinear BVPs. Also, one can see various numerical techniques to obtain the numerical solution of nonlinear BVPs in $[1,2,7,8,11-13,15-17,19,21,24-26]$.

In this paper, first, we apply the quasilinearization technique $[5,19,22]$ to linearize the given nonlinear BVP (1.1) and obtain a sequence of linear BVPs. Each of these linear BVPs is then solved by the numerical scheme obtained from the fractal quintic spline.

\footnotetext{
*Corresponding Author.

Email addresses: balumaths175@gmail.com (N. Balasubramani), mgpp@iitg.ac.in (M. G. P. Prasad), natesan@iitg.ac.in (S. Natesan)

Received: 13.08.2018; Accepted: 11.02.2020
} 
The fractal quintic spline contains a parameter called the scaling factor and the scaling factor is suitably restricted to get the numerical solution of the linearized BVPs.

The rest of the paper is organized as follows: In Section 2, the numerical scheme using the fractal quintic spline has been developed. Convergence analysis of the numerical method is carried out in Section 3. In Section 4, numerical examples are provided to validate the theoretical results.

\section{Numerical scheme}

Here, at the beginning, a brief introduction about the fractal quintic spline is given. Then, we have obtained the relation from the continuity conditions of the fractal quintic spline. This relation is used to derive the numerical scheme of the BVP. Further, we have provided the concept of quasilinearization technique. Finally, we have constructed the numerical scheme to solve the BVP.

\subsection{Fractal quintic spline}

Let $0=x_{0}<x_{1}<\cdots<x_{N}=1$ be the partition of the interval $I=[0,1]$. Let $u(x)$ be the solution of the nonlinear BVP given in (1.1) and $U_{i}$ be the numerical approximate solution of $u\left(x_{i}\right)$. Let $M_{i}$ and $S_{i}$ be the approximations of $u_{x x}\left(x_{i}\right)$ and $u_{x x x x}\left(x_{i}\right)$ respectively.

Barnsley [3] introduced the concept of fractal interpolation function (FIF) with the help of iterated function system (IFS). For more details about IFS and FIFs, one can see the references $[3,4,9,10]$.

Consider the IFS $\left\{I \times \mathbb{R} ; w_{i}(x, y)=\left(L_{i}(x), F_{i}(x, y)\right): i=1,2, \ldots, N\right\}$, where $L_{i}:$ $I \rightarrow I_{i}=\left[x_{i-1}, x_{i}\right]$ defined by $L_{i}(x)=h x+x_{i-1}, \quad x \in I, F_{i}: I \times \mathbb{R} \rightarrow \mathbb{R}$ defined by $F_{i}(x, y)=\alpha y+r_{i}(x), \quad(x, y) \in I \times \mathbb{R}$, with $r_{i}(x)=\mathcal{A}_{i}\left(x-x_{0}\right)^{5}+\mathcal{B}_{i}\left(x-x_{0}\right)^{4}+\mathcal{C}_{i}(x-$ $\left.x_{0}\right)^{3}+\mathcal{D}_{i}\left(x-x_{0}\right)^{2}+\mathcal{E}_{i}\left(x-x_{0}\right)+\mathcal{F}_{i}$ and $\alpha$ is the scaling factor such that $|\alpha|<h^{4}$. For each fixed $i=1,2, \ldots, N$, the function $L_{i}$ satisfy the conditions $L_{i}\left(x_{0}\right)=x_{i-1}, L_{i}\left(x_{N}\right)=x_{i}$. We assume the following conditions on IFS:

$$
\begin{aligned}
& F_{i}\left(x_{0}, U_{0}\right)=U_{i-1}, F_{i}\left(x_{N}, U_{N}\right)=U_{i}, \quad i=1,2, \ldots, N, \\
& F_{i, 1}\left(x_{N}, U_{N, 1}\right)=F_{i+1,1}\left(x_{0}, U_{0,1}\right), i=1,2, \ldots, N-1, \\
& F_{i, 2}\left(x_{0}, M_{0}\right)=M_{i-1}, F_{i, 2}\left(x_{N}, M_{N}\right)=M_{i}, \quad i=1,2, \ldots, N, \\
& F_{i, 3}\left(x_{N}, U_{N, 3}\right)=F_{i+1,3}\left(x_{0}, U_{0,3}\right), i=1,2, \ldots, N-1, \\
& F_{i, 4}\left(x_{0}, S_{0}\right)=S_{i-1}, F_{i, 4}\left(x_{N}, S_{N}\right)=S_{i}, \quad i=1,2, \ldots, N,
\end{aligned}
$$

where

$$
\begin{aligned}
& F_{i, 1}(x, y)=\frac{\alpha y+\left(r_{i}\right)_{x}(x)}{h}, \quad F_{i, 2}(x, y)=\frac{\alpha y+\left(r_{i}\right)_{x x}(x)}{h^{2}}, \\
& F_{i, 3}(x, y)=\frac{\alpha y+\left(r_{i}\right)_{x x x}(x)}{h^{3}}, F_{i, 4}(x, y)=\frac{\alpha y+\left(r_{i}\right)_{x x x x}(x)}{h^{4}}, \\
& U_{0,1}=\frac{\left(r_{1}\right)_{x}\left(x_{0}\right)}{h-\alpha}, \quad U_{N, 1}=\frac{\left(r_{N}\right)_{x}\left(x_{N}\right)}{h-\alpha}, \\
& U_{0,3}=\frac{\left(r_{1}\right)_{x x x}\left(x_{0}\right)}{h^{3}-\alpha}, U_{N, 3}=\frac{\left(r_{N}\right)_{x x x}\left(x_{N}\right)}{h^{3}-\alpha},
\end{aligned}
$$

here $\left(r_{i}\right)_{x}$ represents the derivative of $r_{i}$. Clearly, the IFS $\left\{I \times \mathbb{R} ; w_{i}(x, y)=\left(L_{i}(x), F_{i}(x, y)\right)\right.$ : $i=1,2, \ldots, N\}$ satisfies the conditions for $\mathcal{C}^{4}$-differentiable FIFs $[4,9,10]$.

Let $\mathscr{F}=\left\{\varphi \in \mathcal{C}^{4}(I, \mathbb{R}) \mid \varphi\left(x_{0}\right)=U_{0}, \varphi\left(x_{N}\right)=U_{N}, \varphi_{x x}\left(x_{0}\right)=M_{0}, \varphi_{x x}\left(x_{N}\right)=M_{N}\right.$, $\left.\varphi_{x x x x}\left(x_{0}\right)=S_{0}, \varphi_{x x x x}\left(x_{N}\right)=S_{N}\right\}$. 
Then $(\mathscr{F}, d)$ is a complete metric space where the metric $d$ is induced by the $\mathcal{C}^{4}$-norm on $\mathscr{F}$. Define the Read-Bajraktarević operator $T$ on $(\mathscr{F}, d)$ as

$$
\begin{aligned}
T \varphi\left(L_{i}(x)\right)= & \alpha \varphi(x)+\mathcal{A}_{i}\left(x-x_{0}\right)^{5}+\mathcal{B}_{i}\left(x-x_{0}\right)^{4}+\mathcal{C}_{i}\left(x-x_{0}\right)^{3}+ \\
& \mathcal{D}_{i}\left(x-x_{0}\right)^{2}+\mathcal{E}_{i}\left(x-x_{0}\right)+\mathcal{F}_{i}, \quad x \in\left[x_{0}, x_{N}\right],
\end{aligned}
$$

$i=1,2, \ldots, N$. The map $T$ is a contraction operator, hence it has a unique fixed-point $\Phi$ (say). The fixed-point $\Phi$ satisfies the following functional equation:

$$
\begin{aligned}
\Phi\left(L_{i}(x)\right)= & \alpha \Phi(x)+\mathcal{A}_{i}\left(x-x_{0}\right)^{5}+\mathcal{B}_{i}\left(x-x_{0}\right)^{4}+\mathcal{C}_{i}\left(x-x_{0}\right)^{3}+ \\
& \mathcal{D}_{i}\left(x-x_{0}\right)^{2}+\mathcal{E}_{i}\left(x-x_{0}\right)+\mathcal{F}_{i}, \quad x \in\left[x_{0}, x_{N}\right],
\end{aligned}
$$

$i=1,2, \ldots, N$. The conditions $F_{i}\left(x_{0}, U_{0}\right)=U_{i-1}, F_{i}\left(x_{N}, U_{N}\right)=U_{i}, F_{i, 2}\left(x_{0}, M_{0}\right)=$ $M_{i-1}, F_{i, 2}\left(x_{N}, M_{N}\right)=M_{i}, F_{i, 4}\left(x_{0}, S_{0}\right)=S_{i-1}, F_{i, 4}\left(x_{N}, S_{N}\right)=S_{i}$ are equivalent to the following conditions:

$$
\left\{\begin{array}{l}
\Phi\left(x_{i-1}\right)=U_{i-1}, \Phi\left(x_{i}\right)=U_{i}, \Phi_{x x}\left(x_{i-1}\right)=M_{i-1}, \Phi_{x x}\left(x_{i}\right)=M_{i}, \\
\Phi_{x x x x}\left(x_{i-1}\right)=S_{i-1}, \Phi_{x x x x}\left(x_{i}\right)=S_{i},
\end{array}\right.
$$

respectively. For fractal quintic spline $\Phi$, the constants $\mathcal{A}_{i}, \mathcal{B}_{i}, \mathcal{C}_{i}, \mathcal{D}_{i}, \mathcal{E}_{i}$, and $\mathcal{F}_{i}$ are evaluated by using the conditions given in (2.2) and hence we get

$$
\begin{aligned}
\mathcal{A}_{i}= & \frac{h^{4}}{120}\left[\left(S_{i}-\frac{\alpha}{h^{4}} S_{N}\right)-\left(S_{i-1}-\frac{\alpha}{h^{4}} S_{0}\right)\right], \\
\mathcal{B}_{i}= & \frac{h^{4}}{24}\left(S_{i-1}-\frac{\alpha}{h^{4}} S_{0}\right), \\
\mathcal{C}_{i}= & -\frac{h^{2}}{6}\left(M_{i-1}-\frac{\alpha}{h^{2}} M_{0}\right)+\frac{h^{2}}{6}\left(M_{i}-\frac{\alpha}{h^{2}} M_{N}\right) \\
& -\frac{h^{4}}{12}\left(S_{i-1}-\frac{\alpha}{h^{4}} S_{0}\right)-\frac{h^{4}}{36}\left[\left(S_{i}-\frac{\alpha}{h^{4}} S_{N}\right)-\left(S_{i-1}-\frac{\alpha}{h^{4}} S_{0}\right)\right], \\
\mathcal{D}_{i}= & \frac{h^{2}}{2}\left(M_{i-1}-\frac{\alpha}{h^{2}} M_{0}\right), \\
\mathcal{E}_{i}= & \left(U_{i}-\alpha U_{N}\right)-\left(U_{i-1}-\alpha U_{0}\right)+\frac{8 h^{4}}{360}\left(S_{i-1}-\frac{\alpha}{h^{4}} S_{0}\right) \\
& +\frac{7 h^{4}}{360}\left(S_{i}-\frac{\alpha}{h^{4}} S_{N}\right)-\frac{2 h^{2}}{6}\left(M_{i-1}-\frac{\alpha}{h^{2}} M_{0}\right)-\frac{h^{2}}{6}\left(M_{i}-\frac{\alpha}{h^{2}} M_{N}\right), \\
\mathcal{F}_{i}= & U_{i-1}-\alpha U_{0} .
\end{aligned}
$$

The conditions $F_{i, 1}\left(x_{N}, U_{N, 1}\right)=F_{i+1,1}\left(x_{0}, U_{0,1}\right), i=1,2, \ldots, N-1$ can be reformulated as $\Phi_{x}\left(L_{i}\left(x_{N}\right)\right)=\Phi_{x}\left(L_{i+1}\left(x_{0}\right)\right), i=1,2, \ldots, N-1$. For the continuity of $\Phi_{x}$ at the interior nodes $x_{i}, i=1,2, \ldots, N-1$, we require that $\Phi_{x}\left(x_{i}^{-}\right)=\Phi_{x}\left(x_{i}^{+}\right)$, i.e., $\Phi_{x}\left(L_{i}\left(x_{N}\right)\right)=$ $\Phi_{x}\left(L_{i+1}\left(x_{0}\right)\right), i=1,2, \ldots, N-1$ and this leads to the following:

$$
\alpha \Phi_{x}\left(x_{N}\right)+5 \mathcal{A}_{i}+4 \mathcal{B}_{i}+3 \mathcal{C}_{i}+2 \mathcal{D}_{i}+\mathcal{E}_{i}=\alpha \Phi_{x}\left(x_{0}\right)+\mathcal{E}_{i+1},
$$

substituting the values of $\mathcal{A}_{i}, \mathcal{B}_{i}, \mathcal{C}_{i}, \mathcal{D}_{i}, \mathcal{E}_{i}$, and $\mathcal{E}_{i+1}$, we get

$$
\begin{aligned}
-\frac{15 \alpha}{h^{4}} S_{0} & +7 S_{i-1}+16 S_{i}+7 S_{i+1}-\frac{15 \alpha}{h^{4}} S_{N}=-\frac{360 \alpha}{h^{4}} \Phi_{x}\left(x_{0}\right) \\
& +\frac{360 \alpha}{h^{4}} \Phi_{x}\left(x_{N}\right)-\frac{180 \alpha}{h^{4}} M_{0}+\frac{60}{h^{2}}\left(M_{i+1}+4 M_{i}+M_{i-1}\right)-\frac{180 \alpha}{h^{4}} M_{N} \\
& -\frac{360}{h^{4}}\left(U_{i+1}-2 U_{i}+U_{i-1}\right) .
\end{aligned}
$$

The conditions $F_{i, 3}\left(x_{N}, U_{N, 3}\right)=F_{i+1,3}\left(x_{0}, U_{0,3}\right), i=1,2, \ldots, N-1$ can be reformulated as $\Phi_{x x x}\left(L_{i}\left(x_{N}\right)\right)=\Phi_{x x x}\left(L_{i+1}\left(x_{0}\right)\right), i=1,2, \ldots, N-1$. 
For the continuity of $\Phi_{x x x}$ at the interior nodes $x_{i}, i=1,2, \ldots, N-1$, we need that $\Phi_{x x x}\left(x_{i}^{-}\right)=\Phi_{x x x}\left(x_{i}^{+}\right)$, i.e., $\Phi_{x x x}\left(L_{i}\left(x_{N}\right)\right)=\Phi_{x x x}\left(L_{i+1}\left(x_{0}\right)\right), i=1,2, \ldots, N-1$ and this leads to the following:

$$
\begin{aligned}
-\frac{3 \alpha}{h^{4}} S_{0}+S_{i-1}+4 S_{i}+S_{i+1}-\frac{3 \alpha}{h^{4}} S_{N}= & \frac{6 \alpha}{h^{4}} \Phi_{x x x}\left(x_{0}\right)-\frac{6 \alpha}{h^{4}} \Phi_{x x x}\left(x_{N}\right) \\
& +\frac{6}{h^{2}}\left(M_{i-1}-2 M_{i}+M_{i+1}\right) .
\end{aligned}
$$

Multiplying (2.4) by 7 and subtract the resulting equation from (2.3), we get

$$
\begin{aligned}
& S_{i}=\frac{\alpha}{2 h^{4}}\left(S_{0}+S_{N}\right)+\frac{30 \alpha}{h^{4}}\left(\Phi_{x}\left(x_{0}\right)-\Phi_{x}\left(x_{N}\right)\right)+\frac{7 \alpha}{2 h^{4}}\left(\Phi_{x x x}\left(x_{0}\right)-\Phi_{x x x}\left(x_{N}\right)\right) \\
& +\frac{15 \alpha}{h^{4}}\left(M_{0}+M_{N}\right)-\frac{3}{2 h^{2}}\left(M_{i-1}+18 M_{i}+M_{i+1}\right)+\frac{30}{h^{4}}\left(U_{i+1}-2 U_{i}+U_{i-1}\right) .
\end{aligned}
$$

Substitute $S_{i}$ in (2.4), we obtain

$$
\begin{aligned}
& U_{i-2}+2 U_{i-1}-6 U_{i}+2 U_{i+1}+U_{i+2}=-\frac{\alpha}{2}\left[\Phi_{x x x}\left(x_{0}\right)-\Phi_{x x x}\left(x_{N}\right)\right] \\
& -3 \alpha\left(M_{0}+M_{N}\right)+\frac{h^{2}}{20}\left[M_{i-2}+26 M_{i-1}+66 M_{i}+26 M_{i+1}+M_{i+2}\right] \\
& -6 \alpha\left[\Phi_{x}\left(x_{0}\right)-\Phi_{x}\left(x_{N}\right)\right] .
\end{aligned}
$$

Clearly, the equation (2.5) gives the relation between $U_{i}$ 's and $M_{i}$ 's.

\subsection{Quasilinearization technique}

We use the quasilinearization technique to convert the nonlinear BVP (1.1) into a sequence of linear BVPs. We choose reasonable initial approximation for the function $u(x)$ in $F(x, u(x))$, denote it by $u^{(0)}(x)$, and expand $F(x, u(x))$ around the function $u^{(0)}(x)$ to obtain

$$
\begin{aligned}
F\left(x, u^{(1)}(x)\right)= & F\left(x, u^{(0)}(x)\right)+\left(u^{(1)}(x)-u^{(0)}(x)\right)\left(\frac{\partial F}{\partial u}\right)_{\left(x, u^{(0)}(x)\right)} \\
& +\ldots,
\end{aligned}
$$

and in general, we can write for $r=0,1,2, \ldots(r$ is the iteration index $)$

$$
\begin{aligned}
F\left(x, u^{(r+1)}(x)\right)= & F\left(x, u^{(r)}(x)\right)+\left(u^{(r+1)}(x)-u^{(r)}(x)\right)\left(\frac{\partial F}{\partial u}\right)_{\left(x, u^{(r)}(x)\right)} \\
& +\ldots
\end{aligned}
$$

The nonlinear BVP (1.1) can be written as

$$
\left\{\begin{array}{l}
u_{x x}^{(r+1)}(x)+F\left(x, u^{(r+1)}(x)\right)=0, \quad x \in(0,1) \\
u^{(r+1)}(0)=\eta_{0}, \quad u^{(r+1)}(1)=\eta_{1}
\end{array}\right.
$$

By substituting

$$
F\left(x, u^{(r+1)}(x)\right)=F\left(x, u^{(r)}(x)\right)+\left(u^{(r+1)}(x)-u^{(r)}(x)\right)\left(\frac{\partial F}{\partial u}\right)_{\left(x, u^{(r)}(x)\right)}
$$

in (2.6), we get

$$
\left\{\begin{array}{l}
u_{x x}^{(r+1)}(x)+q^{(r)}(x) u^{(r+1)}(x)=f^{(r)}(x), \quad x \in(0,1), \quad r=0,1, \ldots \\
u^{(r+1)}(0)=\eta_{0}, \quad u^{(r+1)}(1)=\eta_{1},
\end{array}\right.
$$

where

$$
q^{(r)}(x)=\left(\frac{\partial F}{\partial u}\right)_{\left(x, u^{(r)}(x)\right)}, f^{(r)}(x)=u^{(r)}(x)\left(\frac{\partial F}{\partial u}\right)_{\left(x, u^{(r)}(x)\right)}-F\left(x, u^{(r)}(x)\right) .
$$


Thus, we have reduced the nonlinear BVP (1.1) into a sequence of linear BVPs (2.7). Now, our aim is to solve these linear BVPs numerically.

\subsection{Numerical scheme}

Let $|\alpha|<h^{6}$. Let $U_{i}^{(r)}$ be the approximation of $u^{(r)}\left(x_{i}\right)$ and $M_{i}^{(r)}$ be the approximation of $u_{x x}^{(r)}\left(x_{i}\right)$. Now, at $x=x_{i}$, the differential equation (2.7) can be discretized as

$$
M_{i}^{(r+1)}+q_{i}^{(r)} U_{i}^{(r+1)}=f_{i}^{(r)}
$$

where

$$
q_{i}^{(r)}=\left(\frac{\partial F}{\partial u}\right)_{\left(x_{i}, U_{i}^{(r)}\right)}, f_{i}^{(r)}=U_{i}^{(r)}\left(\frac{\partial F}{\partial u}\right)_{\left(x_{i}, U_{i}^{(r)}\right)}-f\left(x_{i}, U_{i}^{(r)}\right) .
$$

Also, the boundary conditions can be discretized as $U_{0}^{(r+1)}=\eta_{0}, \quad U_{N}^{(r+1)}=\eta_{1}$.

Equation (2.5) gives the relation between $U_{i}$ and $M_{i}$ and hence it can be used to derive the numerical scheme for the BVP given in (2.7). Substituting

$$
\begin{aligned}
& \Phi_{x}\left(x_{0}\right)=\frac{U_{1}^{(r+1)}-U_{0}^{(r+1)}}{h}, \Phi_{x}\left(x_{N}\right)=\frac{U_{N}^{(r+1)}-U_{N-1}^{(r+1)}}{h}, \\
& \Phi_{x x x}\left(x_{0}\right)=\frac{-U_{0}^{(r+1)}+3 U_{1}^{(r+1)}-3 U_{2}^{(r+1)}+U_{3}^{(r+1)}}{h^{3}}, \\
& \Phi_{x x x}\left(x_{N}\right)=\frac{U_{N}^{(r+1)}-3 U_{N-1}^{(r+1)}+3 U_{N-2}^{(r+1)}-U_{N-3}^{(r+1)}}{h^{3}}, \\
& M_{i}^{(r+1)}=f_{i}^{(r)}-q_{i}^{(r)} U_{i}^{(r+1)},
\end{aligned}
$$

in (2.5), we get the following system of linear algebraic equations:

$$
\begin{aligned}
& {\left[-\frac{3 \alpha}{2 h^{3}}-\frac{6 \alpha}{h}\right] U_{1}^{(r+1)}+\frac{3 \alpha}{2 h^{3}} U_{2}^{(r+1)}-\frac{\alpha}{2 h^{3}} U_{3}^{(r+1)}+\left[-1-\frac{h^{2}}{20} q_{i-2}^{(r)}\right] U_{i-2}^{(r+1)}} \\
& +\left[-2-\frac{13 h^{2}}{10} q_{i-1}^{(r)}\right] U_{i-1}^{(r+1)}+\left[6-\frac{33 h^{2}}{10} q_{i}^{(r)}\right] U_{i}^{(r+1)} \\
& +\left[-2-\frac{13 h^{2}}{10} q_{i+1}^{(r)}\right] U_{i+1}^{(r+1)}+\left[-1-\frac{h^{2}}{20} q_{i+2}^{(r)}\right] U_{i+2}^{(r+1)}-\frac{\alpha}{2 h^{3}} U_{N-3}^{(r+1)} \\
& +\frac{3 \alpha}{2 h^{3}} U_{N-2}^{(r+1)}+\left[-\frac{3 \alpha}{2 h^{3}}-\frac{6 \alpha}{h}\right] U_{N-1}^{(r+1)}=3 \alpha f_{0}^{(r)}-\frac{h^{2}}{20} f_{i-2}^{(r)}-\frac{13 h^{2}}{10} f_{i-1}^{(r)} \\
& -\frac{33 h^{2}}{10} f_{i}^{(r)}-\frac{13 h^{2}}{10} f_{i+1}^{(r)}-\frac{h^{2}}{20} f_{i+2}^{(r)}+3 \alpha f_{N}^{(r)}-\left[\frac{\alpha}{2 h^{3}}+3 \alpha q_{0}^{(r)}+\frac{6 \alpha}{h}\right] \eta_{0} \\
& -\left[\frac{\alpha}{2 h^{3}}+3 \alpha q_{N}^{(r)}+\frac{6 \alpha}{h}\right] \eta_{1}, i=2,3, \ldots, N-2 .
\end{aligned}
$$

In the system of equations given in $(2.8)$ we have $(N-3)$ equations with $(N-1)$ unknowns $U_{1}^{(r+1)}, U_{2}^{(r+1)}, \ldots, U_{N-1}^{(r+1)}$. In order to determine a unique solution, we require two more equations. For that we use the following procedure.

Firstly, we determine the truncation error

$$
\begin{aligned}
& T_{1}^{(r)}(h)=\left[\frac{\alpha}{2 h^{3}}+3 \alpha q^{(r)}\left(x_{0}\right)+\frac{6 \alpha}{h}\right] u^{(r+1)}\left(x_{0}\right)+\left[-\frac{3 \alpha}{2 h^{3}}-\frac{6 \alpha}{h}\right] u^{(r+1)}\left(x_{1}\right) \\
& +\frac{3 \alpha}{2 h^{3}} u^{(r+1)}\left(x_{2}\right)-\frac{\alpha}{2 h^{3}} u^{(r+1)}\left(x_{3}\right)-\frac{\alpha}{2 h^{3}} u^{(r+1)}\left(x_{N-3}\right)+\frac{3 \alpha}{2 h^{3}} u^{(r+1)}\left(x_{N-2}\right) \\
& +\left[-\frac{3 \alpha}{2 h^{3}}-\frac{6 \alpha}{h}\right] u^{(r+1)}\left(x_{N-1}\right)+\left[\frac{\alpha}{2 h^{3}}+3 \alpha q^{(r)}\left(x_{N}\right)+\frac{6 \alpha}{h}\right] u^{(r+1)}\left(x_{N}\right) \\
& -3 \alpha f^{(r)}\left(x_{0}\right)-3 \alpha f^{(r)}\left(x_{N}\right)+\sum_{i=0}^{3}\left(c_{i} u^{(r+1)}\left(x_{i}\right)+d_{i} h^{2} u_{x x}^{(r+1)}\left(x_{i}\right)\right) .
\end{aligned}
$$


Substituting $f^{(r)}\left(x_{i}\right)=u_{x x}^{(r+1)}\left(x_{i}\right)+q^{(r)}\left(x_{i}\right) u^{(r+1)}\left(x_{i}\right)$ in (2.9), we get

$$
\begin{aligned}
& T_{1}^{(r)}(h)=-\frac{\alpha}{2}\left[\frac{-u^{(r+1)}\left(x_{0}\right)+3 u^{(r+1)}\left(x_{1}\right)-3 u^{(r+1)}\left(x_{2}\right)+u^{(r+1)}\left(x_{3}\right)}{h^{3}}\right] \\
& +\frac{\alpha}{2}\left[\frac{u^{(r+1)}\left(x_{N}\right)-3 u^{(r+1)}\left(x_{N-1}\right)+3 u^{(r+1)}\left(x_{N-2}\right)-u^{(r+1)}\left(x_{N-3}\right)}{h^{3}}\right] \\
& -6 \alpha\left[\frac{-u^{(r+1)}\left(x_{0}\right)+u^{(r+1)}\left(x_{1}\right)}{h}\right]+6 \alpha\left[\frac{u^{(r+1)}\left(x_{N}\right)-u^{(r+1)}\left(x_{N-1}\right)}{h}\right] \\
& -3 \alpha u_{x x}^{(r+1)}\left(x_{0}\right)-3 \alpha u_{x x}^{(r+1)}\left(x_{N}\right)+\sum_{i=0}^{3}\left(c_{i} u^{(r+1)}\left(x_{i}\right)+d_{i} h^{2} u_{x x}^{(r+1)}\left(x_{i}\right)\right) .
\end{aligned}
$$

It can be seen that

$$
\begin{aligned}
& \frac{-u^{(r+1)}\left(x_{0}\right)+3 u^{(r+1)}\left(x_{1}\right)-3 u^{(r+1)}\left(x_{2}\right)+u^{(r+1)}\left(x_{3}\right)}{h^{3}}=u_{x x x}^{(r+1)}\left(x_{0}\right)+\mathcal{O}(h), \\
& \frac{-u^{(r+1)}\left(x_{0}\right)+u^{(r+1)}\left(x_{1}\right)}{h}=u_{x}^{(r+1)}\left(x_{0}\right)+\mathcal{O}(h), \\
& \frac{u^{(r+1)}\left(x_{N}\right)-3 u^{(r+1)}\left(x_{N-1}\right)+3 u^{(r+1)}\left(x_{N-2}\right)-u^{(r+1)}\left(x_{N-3}\right)}{h^{3}}=u_{x x x}^{(r+1)}\left(x_{N}\right)+\mathcal{O}(h), \\
& \frac{u^{(r+1)}\left(x_{N}\right)-u^{(r+1)}\left(x_{N-1}\right)}{h}=u_{x}^{(r+1)}\left(x_{N}\right)+\mathcal{O}(h) .
\end{aligned}
$$

Hence, we have

$$
\begin{aligned}
& T_{1}^{(r)}(h)=-\frac{\alpha}{2}\left[u_{x x x}^{(r+1)}\left(x_{0}\right)+\mathcal{O}(h)\right]+\frac{\alpha}{2}\left[u_{x x x}^{(r+1)}\left(x_{N}\right)+\mathcal{O}(h)\right] \\
& -6 \alpha\left[u_{x}^{(r+1)}\left(x_{0}\right)+\mathcal{O}(h)\right]+6 \alpha\left[u_{x}^{(r+1)}\left(x_{N}\right)+\mathcal{O}(h)\right] \\
& -3 \alpha u_{x x}^{(r+1)}\left(x_{0}\right)-3 \alpha u_{x x}^{(r+1)}\left(x_{N}\right)+\sum_{i=0}^{3}\left(c_{i} u^{(r+1)}\left(x_{i}\right)+d_{i} h^{2} u_{x x}^{(r+1)}\left(x_{i}\right)\right) .
\end{aligned}
$$

Our aim is to get $T_{1}^{(r)}(h)=\mathcal{O}\left(h^{6}\right)$. We write

$$
T_{1}^{(r)}(h)=T_{\alpha}^{(r)}(h)+T_{*}^{(r)}(h),
$$

where

$$
\begin{aligned}
& T_{\alpha}^{(r)}(h)=-\frac{\alpha}{2}\left[u_{x x x}^{(r+1)}\left(x_{0}\right)+\mathcal{O}(h)\right]+\frac{\alpha}{2}\left[u_{x x x}^{(r+1)}\left(x_{N}\right)+\mathcal{O}(h)\right] \\
& -6 \alpha\left[u_{x}^{(r+1)}\left(x_{0}\right)+\mathcal{O}(h)\right]+6 \alpha\left[u_{x}^{(r+1)}\left(x_{N}\right)+\mathcal{O}(h)\right] \\
& -3 \alpha u_{x x}^{(r+1)}\left(x_{0}\right)-3 \alpha u_{x x}^{(r+1)}\left(x_{N}\right)
\end{aligned}
$$

and

$$
T_{*}^{(r)}(h)=\sum_{i=0}^{3}\left(c_{i} u^{(r+1)}\left(x_{i}\right)+d_{i} h^{2} u_{x x}^{(r+1)}\left(x_{i}\right)\right) .
$$

Since $|\alpha|<h^{6}$, we have $T_{\alpha}^{(r)}(h)=\mathcal{O}\left(h^{6}\right)$. Next, our aim is to choose $c_{0}, c_{1}, c_{2}, c_{3}, d_{0}, d_{1}$, $d_{2}$, and $d_{3}$ such that $T_{*}^{(r)}(h)=\mathcal{O}\left(h^{6}\right)$. By using the Taylor series expansions for $u^{(r+1)}\left(x_{0}\right)$, $u^{(r+1)}\left(x_{2}\right), u^{(r+1)}\left(x_{3}\right), u_{x x}^{(r+1)}\left(x_{0}\right), u_{x x}^{(r+1)}\left(x_{2}\right)$, and $u_{x x}^{(r+1)}\left(x_{3}\right)$ about the point $x=x_{1}$, we get 


$$
\begin{aligned}
T_{*}^{(r)}(h)= & {\left[c_{0}+c_{1}+c_{2}+c_{3}\right] u^{(r+1)}\left(x_{1}\right)+\left[-c_{0}+c_{2}+2 c_{3}\right] h u_{x}^{(r+1)}\left(x_{1}\right) } \\
& +\left[\frac{1}{2 !} c_{0}+\frac{1}{2 !} c_{2}+\frac{2^{2}}{2 !} c_{3}+d_{0}+d_{1}+d_{2}+d_{3}\right] h^{2} u_{x x}^{(r+1)}\left(x_{1}\right) \\
& +\left[-\frac{1}{3 !} c_{0}+\frac{1}{3 !} c_{2}+\frac{2^{3}}{3 !} c_{3}-d_{0}+d_{2}+2 d_{3}\right] h^{3} u_{x x x}^{(r+1)}\left(x_{1}\right) \\
& +\left[\frac{1}{4 !} c_{0}+\frac{1}{4 !} c_{2}+\frac{2^{4}}{4 !} c_{3}+\frac{1}{2 !} d_{0}+\frac{1}{2 !} d_{2}+\frac{2^{2}}{2 !} d_{3}\right] h^{4} u_{x x x x}^{(r+1)}\left(x_{1}\right) \\
& +\left[-\frac{1}{5 !} c_{0}+\frac{1}{5 !} c_{2}+\frac{2^{5}}{5 !} c_{3}-\frac{1}{3 !} d_{0}+\frac{1}{3 !} d_{2}+\frac{2^{3}}{3 !} d_{3}\right] h^{5} u_{x x x x x}^{(r+1)}\left(x_{1}\right) \\
& +\left[\frac{1}{6 !} c_{0}+\frac{1}{6 !} c_{2}+\frac{2^{6}}{6 !} c_{3}+\frac{1}{4 !} d_{0}+\frac{1}{4 !} d_{2}+\frac{2^{4}}{4 !} d_{3}\right] h^{6} u_{x x x x x x}^{(r+1)}\left(x_{1}\right) \\
& +\mathcal{O}\left(h^{7}\right) .
\end{aligned}
$$

Hence to get $T_{*}^{(r)}(h)=\mathcal{O}\left(h^{6}\right)$, we need

$$
\begin{aligned}
& c_{0}+c_{1}+c_{2}+c_{3}=0, \quad-c_{0}+c_{2}+2 c_{3}=0, \\
& \frac{1}{2 !} c_{0}+\frac{1}{2 !} c_{2}+\frac{2^{2}}{2 !} c_{3}+d_{0}+d_{1}+d_{2}+d_{3}=0, \\
& -\frac{1}{3 !} c_{0}+\frac{1}{3 !} c_{2}+\frac{2^{3}}{3 !} c_{3}-d_{0}+d_{2}+2 d_{3}=0, \\
& \frac{1}{4 !} c_{0}+\frac{1}{4 !} c_{2}+\frac{2^{4}}{4 !} c_{3}+\frac{1}{2 !} d_{0}+\frac{1}{2 !} d_{2}+\frac{2^{2}}{2 !} d_{3}=0, \\
& -\frac{1}{5 !} c_{0}+\frac{1}{5 !} c_{2}+\frac{2^{5}}{5 !} c_{3}-\frac{1}{3 !} d_{0}+\frac{1}{3 !} d_{2}+\frac{2^{3}}{3 !} d_{3}=0 .
\end{aligned}
$$

Here, we have 6 equations with 8 unknowns $c_{0}, c_{1}, c_{2}, c_{3}, d_{0}, d_{1}, d_{2}$, and $d_{3}$. Let us fix two unknowns $c_{1}$ and $c_{2}$ as $c_{1}=7$ and $c_{2}=-2$. Hence we can solve the system of equations and obtain $c_{0}=-4, c_{3}=-1, d_{0}=\frac{1}{3}, d_{1}=\frac{41}{12}, d_{2}=\frac{7}{6}$, and $d_{3}=\frac{1}{12}$. The constants $c_{1}=7$ and $c_{2}=-2$ are carefully chosen so that the matrix $B^{(r)}$ (given below) would be a monotone matrix.

It can be seen that, by substituting $c_{0}=-4, c_{1}=7, c_{2}=-2, c_{3}=-1, d_{0}=\frac{1}{3}$, $d_{1}=\frac{41}{12}, d_{2}=\frac{7}{6}$, and $d_{3}=\frac{1}{12}$, we get $T_{*}^{(r)}(h)=\frac{h^{6}}{48} u_{x x x x x x}^{(r+1)}\left(x_{1}\right)+\mathcal{O}\left(h^{7}\right)$. Hence we have $T_{*}^{(r)}(h)=\mathcal{O}\left(h^{6}\right)$. Thus, by the assumption $|\alpha|<h^{6}, c_{0}=-4, c_{1}=7, c_{2}=-2, c_{3}=-1$, $d_{0}=\frac{1}{3}, d_{1}=\frac{41}{12}, d_{2}=\frac{7}{6}$, and $d_{3}=\frac{1}{12}$, we get

$$
\begin{aligned}
T_{1}^{(r)}(h)= & {\left[\frac{\alpha}{2 h^{3}}+3 \alpha q^{(r)}\left(x_{0}\right)+\frac{6 \alpha}{h}\right] u^{(r+1)}\left(x_{0}\right)+\left[-\frac{3 \alpha}{2 h^{3}}-\frac{6 \alpha}{h}\right] u^{(r+1)}\left(x_{1}\right) } \\
& +\frac{3 \alpha}{2 h^{3}} u^{(r+1)}\left(x_{2}\right)-\frac{\alpha}{2 h^{3}} u^{(r+1)}\left(x_{3}\right)-\frac{\alpha}{2 h^{3}} u^{(r+1)}\left(x_{N-3}\right)+\frac{3 \alpha}{2 h^{3}} u^{(r+1)}\left(x_{N-2}\right) \\
& +\left[-\frac{3 \alpha}{2 h^{3}}-\frac{6 \alpha}{h}\right] u^{(r+1)}\left(x_{N-1}\right)+\left[\frac{\alpha}{2 h^{3}}+3 \alpha q^{(r)}\left(x_{N}\right)+\frac{6 \alpha}{h}\right] u^{(r+1)}\left(x_{N}\right) \\
& -3 \alpha f^{(r)}\left(x_{0}\right)-3 \alpha f^{(r)}\left(x_{N}\right)-4 u^{(r+1)}\left(x_{0}\right)+7 u^{(r+1)}\left(x_{1}\right) \\
& -2 u^{(r+1)}\left(x_{2}\right)-u^{(r+1)}\left(x_{3}\right)+\frac{1}{3} h^{2} u_{x x}^{(r+1)}\left(x_{0}\right)+\frac{41}{12} h^{2} u_{x x}^{(r+1)}\left(x_{1}\right) \\
& +\frac{7}{6} h^{2} u_{x x}^{(r+1)}\left(x_{2}\right)+\frac{1}{12} h^{2} u_{x x}^{(r+1)}\left(x_{3}\right) .
\end{aligned}
$$

When $h \rightarrow 0$, we have $\left|T_{1}^{(r)}(h)\right| \leq K_{1}^{(r)} h^{6}$, where $K_{1}^{(r)}$ is a constant. Thus $T_{1}^{(r)}(h)=\mathcal{O}\left(h^{6}\right)$. Hence from $(2.10)$, replacing the original values $u^{(r)}\left(x_{i}\right)$ and $u_{x x}^{(r)}\left(x_{i}\right)$ by the approximate 
values $U_{i}^{(r)}$ and $M_{i}^{(r)}$, respectively, we get the difference equation (associated with the truncation error $\left.T_{1}^{(r)}(h)\right)$ as

$$
\begin{aligned}
& {\left[7-\frac{41 h^{2}}{12} q_{1}^{(r)}-\frac{3 \alpha}{2 h^{3}}-\frac{6 \alpha}{h}\right] U_{1}^{(r+1)}+\left[-2-\frac{7 h^{2}}{6} q_{2}^{(r)}+\frac{3 \alpha}{2 h^{3}}\right] U_{2}^{(r+1)}} \\
& +\left[-1-\frac{h^{2}}{12} q_{3}^{(r)}-\frac{\alpha}{2 h^{3}}\right] U_{3}^{(r+1)}-\frac{\alpha}{2 h^{3}} U_{N-3}^{(r+1)}+\frac{3 \alpha}{2 h^{3}} U_{N-2}^{(r+1)} \\
& +\left[-\frac{3 \alpha}{2 h^{3}}-\frac{6 \alpha}{h}\right] U_{N-1}^{(r+1)}=3 \alpha f_{0}^{(r)}-\frac{h^{2}}{3} f_{0}^{(r)}-\frac{41 h^{2}}{12} f_{1}^{(r)}-\frac{7 h^{2}}{6} f_{2}^{(r)} \\
& -\frac{h^{2}}{12} f_{3}^{(r)}+3 \alpha f_{N}^{(r)}-\left[-4-\frac{h^{2}}{3} f_{0}^{(r)}+\frac{\alpha}{2 h^{3}}+3 \alpha q_{0}^{(r)}+\frac{6 \alpha}{h}\right] \eta_{0} \\
& -\left[\frac{\alpha}{2 h^{3}}+3 \alpha q_{N}^{(r)}+\frac{6 \alpha}{h}\right] \eta_{1} .
\end{aligned}
$$

Similarly, we can define the truncation error

$$
\begin{aligned}
& T_{N-1}^{(r)}(h)=\left[\frac{\alpha}{2 h^{3}}+3 \alpha q^{(r)}\left(x_{0}\right)+\frac{6 \alpha}{h}\right] u^{(r+1)}\left(x_{0}\right)+\left[-\frac{3 \alpha}{2 h^{3}}-\frac{6 \alpha}{h}\right] u^{(r+1)}\left(x_{1}\right) \\
& +\frac{3 \alpha}{2 h^{3}} u^{(r+1)}\left(x_{2}\right)-\frac{\alpha}{2 h^{3}} u^{(r+1)}\left(x_{3}\right)-\frac{\alpha}{2 h^{3}} u^{(r+1)}\left(x_{N-3}\right) \\
& +\frac{3 \alpha}{2 h^{3}} u^{(r+1)}\left(x_{N-2}\right)+\left[-\frac{3 \alpha}{2 h^{3}}-\frac{6 \alpha}{h}\right] u^{(r+1)}\left(x_{N-1}\right)+\left[\frac{\alpha}{2 h^{3}}\right. \\
& \left.+3 \alpha q^{(r)}\left(x_{N}\right)+\frac{6 \alpha}{h}\right] u^{(r+1)}\left(x_{N}\right)-3 \alpha f^{(r)}\left(x_{0}\right)-3 \alpha f^{(r)}\left(x_{N}\right) \\
& \quad+\sum_{i=N-3}^{N}\left(c_{i} u^{(r+1)}\left(x_{i}\right)+d_{i} h^{2} u_{x x}^{(r+1)}\left(x_{i}\right)\right) .
\end{aligned}
$$

By the assumption $|\alpha|<h^{6}, c_{N}=-4, c_{N-1}=7, c_{N-2}=-2, c_{N-3}=-1, d_{N}=\frac{1}{3}$, $d_{N-1}=\frac{41}{12}, d_{N-2}=\frac{7}{6}$, and $d_{N-3}=\frac{1}{12}$, we get $T_{N-1}^{(r)}(h)=\mathcal{O}\left(h^{6}\right)$, that is, when $h \rightarrow 0$, we have $\left|T_{N-1}^{(r)}(h)\right| \leq K_{N-1}^{(r)} h^{6}$, where $K_{N-1}^{(r)}$ is a constant.

Hence, we get the difference equation (associated with $\left.T_{N-1}^{(r)}(h)\right)$ as follows:

$$
\begin{aligned}
& {\left[-\frac{3 \alpha}{2 h^{3}}-\frac{6 \alpha}{h}\right] U_{1}^{(r+1)}+\frac{3 \alpha}{2 h^{3}} U_{2}^{(r+1)}-\frac{\alpha}{2 h^{3}} U_{3}^{(r+1)}+\left[-1-\frac{h^{2}}{12} q_{N-3}^{(r)}\right.} \\
& \left.-\frac{\alpha}{2 h^{3}}\right] U_{N-3}^{(r+1)}+\left[-2-\frac{7 h^{2}}{6} q_{N-2}^{(r)}+\frac{3 \alpha}{2 h^{3}}\right] U_{N-2}^{(r+1)}+\left[7-\frac{41 h^{2}}{12} q_{N-1}^{(r)}\right. \\
& \left.-\frac{3 \alpha}{2 h^{3}}-\frac{6 \alpha}{h}\right] U_{N-1}^{(r+1)}=3 \alpha f_{0}^{(r)}-\frac{h^{2}}{3} f_{N}^{(r)}-\frac{41 h^{2}}{12} f_{N-1}^{(r)}-\frac{7 h^{2}}{6} f_{N-2}^{(r)} \\
& -\frac{h^{2}}{12} f_{N-3}^{(r)}+3 \alpha f_{N}^{(r)}-\left[\frac{\alpha}{2 h^{3}}+3 \alpha q_{0}^{(r)}+\frac{6 \alpha}{h}\right] \eta_{0} \\
& -\left[-4-\frac{h^{2}}{3} f_{N}^{(r)}+\frac{\alpha}{2 h^{3}}+3 \alpha q_{N}^{(r)}+\frac{6 \alpha}{h}\right] \eta_{1} .
\end{aligned}
$$

The system given in (2.11), (2.8), and (2.12) gives the approximations $U_{1}^{(r+1)}, U_{2}^{(r+1)}, \ldots$, $U_{N-1}^{(r+1)}$.

Remark 2.1. The system corresponding to the quintic spline can be obtained by taking $\alpha=0$ in (2.11), (2.8), and (2.12). 


\section{Convergence analysis}

In this section, first, we derive the truncation error corresponding to the system of equations given in (2.11), (2.8), and (2.12). Then, we obtain the error bound of the proposed numerical method.

\subsection{Truncation error}

We define the truncation error $T_{i}^{(r)}(h), i=2,3, \ldots, N-2$, associated with the equations given in (2.8) as

$$
\begin{aligned}
& T_{i}^{(r)}(h)=\left[\frac{\alpha}{2 h^{3}}+3 \alpha q^{(r)}\left(x_{0}\right)+\frac{6 \alpha}{h}\right] u^{(r+1)}\left(x_{0}\right)+\left[-\frac{3 \alpha}{2 h^{3}}-\frac{6 \alpha}{h}\right] u^{(r+1)}\left(x_{1}\right) \\
& +\frac{3 \alpha}{2 h^{3}} u^{(r+1)}\left(x_{2}\right)-\frac{\alpha}{2 h^{3}} u^{(r+1)}\left(x_{3}\right)+\left[-1-\frac{h^{2}}{20} q^{(r)}\left(x_{i-2}\right)\right] u^{(r+1)}\left(x_{i-2}\right) \\
& +\left[-2-\frac{13 h^{2}}{10} q^{(r)}\left(x_{i-1}\right)\right] u^{(r+1)}\left(x_{i-1}\right)+\left[6-\frac{33 h^{2}}{10} q^{(r)}\left(x_{i}\right)\right] u^{(r+1)}\left(x_{i}\right) \\
& +\left[-2-\frac{13 h^{2}}{10} q^{(r)}\left(x_{i+1}\right)\right] u^{(r+1)}\left(x_{i+1}\right)+\left[-\frac{h^{2}}{20} q^{(r)}\left(x_{i+2}\right)\right. \\
& -1] u^{(r+1)}\left(x_{i+2}\right)-\frac{\alpha}{2 h^{3}} u^{(r+1)}\left(x_{N-3}\right)+\frac{3 \alpha}{2 h^{3}} u^{(r+1)}\left(x_{N-2}\right) \\
& +\left[-\frac{3 \alpha}{2 h^{3}}-\frac{6 \alpha}{h}\right] u^{(r+1)}\left(x_{N-1}\right)+\left[\frac{\alpha}{2 h^{3}}+3 \alpha q^{(r)}\left(x_{N}\right)+\frac{6 \alpha}{h}\right] u^{(r+1)}\left(x_{N}\right) \\
& -3 \alpha f^{(r)}\left(x_{0}\right)+\frac{h^{2}}{20} f^{(r)}\left(x_{i-2}\right)+\frac{13 h^{2}}{10} f^{(r)}\left(x_{i-1}\right)+\frac{33 h^{2}}{10} f^{(r)}\left(x_{i}\right) \\
& +\frac{13 h^{2}}{10} f^{(r)}\left(x_{i+1}\right)+\frac{h^{2}}{20} f^{(r)}\left(x_{i+2}\right)-3 \alpha f^{(r)}\left(x_{N}\right) .
\end{aligned}
$$

Substituting $f^{(r)}\left(x_{i}\right)=u_{x x}^{(r+1)}\left(x_{i}\right)+q^{(r)}\left(x_{i}\right) u^{(r+1)}\left(x_{i}\right)$ in (3.1), we get

$$
\begin{aligned}
& T_{i}^{(r)}(h)=-\frac{\alpha}{2}\left[\frac{-u^{(r+1)}\left(x_{0}\right)+3 u^{(r+1)}\left(x_{1}\right)-3 u^{(r+1)}\left(x_{2}\right)+u^{(r+1)}\left(x_{3}\right)}{h^{3}}\right] \\
& +\frac{\alpha}{2}\left[\frac{u^{(r+1)}\left(x_{N}\right)-3 u^{(r+1)}\left(x_{N-1}\right)+3 u^{(r+1)}\left(x_{N-2}\right)-u^{(r+1)}\left(x_{N-3}\right)}{h^{3}}\right] \\
& -6 \alpha\left[\frac{-u^{(r+1)}\left(x_{0}\right)+u^{(r+1)}\left(x_{1}\right)}{h}\right]+6 \alpha\left[\frac{u^{(r+1)}\left(x_{N}\right)-u^{(r+1)}\left(x_{N-1}\right)}{h}\right] \\
& -3 \alpha u_{x x}^{(r+1)}\left(x_{0}\right)-3 \alpha u_{x x}^{(r+1)}\left(x_{N}\right)-u^{(r+1)}\left(x_{i-2}\right)-2 u^{(r+1)}\left(x_{i-1}\right) \\
& +6 u^{(r+1)}\left(x_{i}\right)-2 u^{(r+1)}\left(x_{i+1}\right)-u^{(r+1)}\left(x_{i+2}\right)+\frac{h^{2}}{20} u_{x x}^{(r+1)}\left(x_{i-2}\right) \\
& +\frac{13 h^{2}}{10} u_{x x}^{(r+1)}\left(x_{i-1}\right)+\frac{33 h^{2}}{10} u_{x x}^{(r+1)}\left(x_{i}\right)+\frac{13 h^{2}}{10} u_{x x}^{(r+1)}\left(x_{i+1}\right) \\
& +\frac{h^{2}}{20} u_{x x}^{(r+1)}\left(x_{i+2}\right) .
\end{aligned}
$$

After further simplification, we obtain that

$$
\begin{aligned}
& T_{i}^{(r)}(h)=-\frac{\alpha}{2}\left[u_{x x x}^{(r+1)}\left(x_{0}\right)+\mathcal{O}(h)\right]+\frac{\alpha}{2}\left[u_{x x x}^{(r+1)}\left(x_{N}\right)+\mathcal{O}(h)\right] \\
& -6 \alpha\left[u_{x}^{(r+1)}\left(x_{0}\right)+\mathcal{O}(h)\right]+6 \alpha\left[u_{x}^{(r+1)}\left(x_{N}\right)+\mathcal{O}(h)\right] \\
& -3 \alpha u_{x x}^{(r+1)}\left(x_{0}\right)-3 \alpha u_{x x}^{(r+1)}\left(x_{N}\right)-\frac{h^{6}}{120} u_{x x x x x x}^{(r+1)}\left(x_{i}\right)+\mathcal{O}\left(h^{7}\right),
\end{aligned}
$$


$i=2,3, \ldots, N-2$. When $h \rightarrow 0$, we have $\left|T_{i}^{(r)}(h)\right| \leq K_{i}^{(r)} h^{6}$ where $K_{i}^{(r)}$ is a constant. It can be seen that $T_{i}^{(r)}(h)=\mathcal{O}\left(h^{6}\right), i=2,3, \ldots, N-2$.

Remark 3.1. The system given in (2.11), (2.8), and (2.12) can be written in the form $A^{(r)} U^{(r+1)}=d^{(r)}$, where

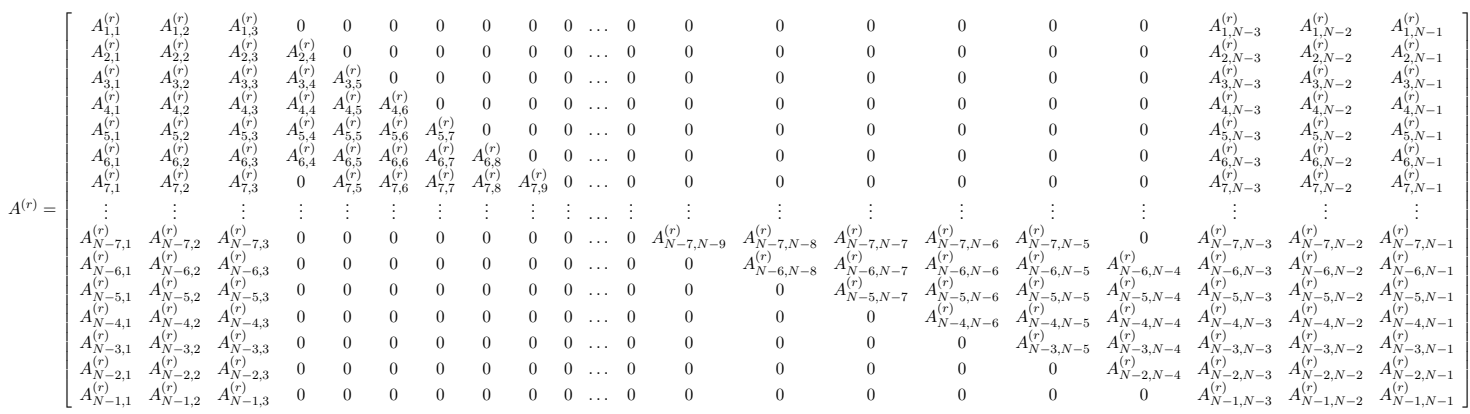

$A_{i, j}^{(r)}$ is the coefficient of $U_{j}^{(r+1)}, U^{(r+1)}=\left(U_{1}^{(r+1)}, U_{2}^{(r+1)}, \ldots, U_{N-1}^{(r+1)}\right)^{T}, d^{(r)}=\left(d_{1}^{(r)}, d_{2}^{(r)}\right.$, $\left.\ldots, d_{N-1}^{(r)}\right)^{T}, d_{i}^{(r)}$ is the right-hand side of the system.

Let $B^{(r)}$ be the matrix corresponding to the quintic spline system given in Remark 2.1. Note that, $\left\|A^{(r)}-B^{(r)}\right\|_{\infty}=\max _{i} \sum_{j=1}^{N-1}\left|A_{i, j}^{(r)}-B_{i, j}^{(r)}\right|$. Thus, we get

$$
\left\|A^{(r)}-B^{(r)}\right\|_{\infty}=2\left|-\frac{3 \alpha}{2 h^{3}}-\frac{6 \alpha}{h}\right|+2\left|\frac{3 \alpha}{2 h^{3}}\right|+2\left|-\frac{\alpha}{2 h^{3}}\right| .
$$

Theorem 3.2 ([6]). Let $Q_{1}$ and $Q_{2}$ be any two $n \times n$ matrices, and let $\|$.$\| be any matrix$ norm. Then the eigenvalues of $Q_{1}$ and $Q_{2}$ can be enumerated as $\lambda_{1}, \lambda_{2}, \ldots, \lambda_{n}$ and $\mu_{1}$, $\mu_{2}, \ldots, \mu_{n}$ in such a way that

$$
\max _{i}\left|\lambda_{i}-\mu_{i}\right| \leq 2^{\frac{2 n-1}{n}} n^{\frac{1}{n}}(2 P)^{\frac{n-1}{n}}\left\|Q_{1}-Q_{2}\right\|^{\frac{1}{n}},
$$

where $P=\max \left\{\left\|Q_{1}\right\|,\left\|Q_{2}\right\|\right\}$.

In our case, we take the matrices $A^{(r)}=Q_{1}, B^{(r)}=Q_{2}, n=N-1,\|\cdot\|_{\infty}$ norm in the previous theorem, then from (3.2), we get

$$
\max _{i}\left|\lambda_{i}^{(r)}-\mu_{i}^{(r)}\right| \leq 2^{\frac{2 N-3}{N-1}}(N-1)^{\frac{1}{N-1}}\left(2 P^{(r)}\right)^{\frac{N-2}{N-1}}\left\|A^{(r)}-B^{(r)}\right\|_{\infty}^{\frac{1}{N-1}},
$$

where $P^{(r)}=\max \left\{\left\|A^{(r)}\right\|_{\infty},\left\|B^{(r)}\right\|_{\infty}\right\}, \lambda_{i}^{(r)}$ and $\mu_{i}^{(r)}, i=1,2, \ldots, N-1$ are the eigenvalues of $A^{(r)}$ and $B^{(r)}$ respectively.

Note that, when $h$ is sufficiently small, $B^{(r)}$ becomes irreducible, $B_{i, i}^{(r)}>0, B_{i, j}^{(r)} \leq 0$, $i \neq j, i, j=1,2, \ldots, N-1$, and the row sums give

$$
\begin{aligned}
& \mathcal{R}_{1}^{(r)}=4-\frac{41 h^{2}}{12} q_{1}^{(r)}-\frac{7 h^{2}}{6} q_{2}^{(r)}-\frac{h^{2}}{12} q_{3}^{(r)}>0, \\
& \mathcal{R}_{2}^{(r)}=1-\frac{13 h^{2}}{10} q_{1}^{(r)}-\frac{33 h^{2}}{10} q_{2}^{(r)}-\frac{13 h^{2}}{10} q_{3}^{(r)}-\frac{h^{2}}{20} q_{4}^{(r)}>0, \\
& \mathcal{R}_{i}^{(r)}=-\frac{h^{2}}{20} q_{i-2}^{(r)}-\frac{13 h^{2}}{10} q_{i-1}^{(r)}-\frac{33 h^{2}}{10} q_{i}^{(r)}-\frac{13 h^{2}}{10} q_{i+1}^{(r)}-\frac{h^{2}}{20} q_{i+2}^{(r)}>0, \\
& i=3,4, \ldots, N-3, \\
& \mathcal{R}_{N-2}^{(r)}=1-\frac{13 h^{2}}{10} q_{N-1}^{(r)}-\frac{33 h^{2}}{10} q_{N-2}^{(r)}-\frac{13 h^{2}}{10} q_{N-3}^{(r)}-\frac{h^{2}}{20} q_{N-4}^{(r)}>0, \\
& \mathcal{R}_{N-1}^{(r)}=4-\frac{41 h^{2}}{12} q_{N-1}^{(r)}-\frac{7 h^{2}}{6} q_{N-2}^{(r)}-\frac{h^{2}}{12} q_{N-3}^{(r)}>0 .
\end{aligned}
$$


Hence $B^{(r)}$ is a monotone matrix [18]. Thus, $B^{(r)^{-1}}$ exist and the eigenvalues $\mu_{i}^{(r)}, i=$ $1,2, \ldots, N-1$ of $B^{(r)}$ are non-zero when $h$ is sufficiently small. Thus, once we take sufficiently small $h$ (which makes $B^{(r)}$ is a monotone matrix), then $\alpha$ can vary in the region $\left(-h^{6}, h^{6}\right)$. Now we can choose $\alpha$ to satisfy the following conditions:

- $A^{(r)}$ is invertible because $\left\|A^{(r)}-B^{(r)}\right\|_{\infty}=2\left|-\frac{3 \alpha}{2 h^{3}}-\frac{6 \alpha}{h}\right|+2\left|\frac{3 \alpha}{2 h^{3}}\right|+2\left|-\frac{\alpha}{2 h^{3}}\right|$ and from (3.3), it can been seen that, the eigenvalues of $A^{(r)}$ are non-zero when $\alpha$ is sufficiently small.

- Since $\mathcal{R}_{i}^{(r)}>0, i=1,2, \ldots, N-1$, the row sums of $A^{(r)}$,

$\mathcal{S}_{i}^{(r)}=\mathcal{R}_{i}^{(r)}-\frac{12 \alpha}{h}-\frac{\alpha}{h^{3}}>0, i=1,2, \ldots, N-1$,

when $\alpha$ is sufficiently small.

For sufficiently small $h$ (which makes $B^{(r)}$ is monotone) and for sufficiently small $\alpha \in$ $\left(-h^{6}, h^{6}\right)$ (which makes $A^{(r)}$ is invertible and row sums of $A^{(r)}$ is positive), we obtain the following error bound.

\subsection{Error bound}

The system (2.11), (2.8), and (2.12) with exact solutions can be written as

$$
A^{(r)} \bar{u}^{(r+1)}=d^{(r)}+T^{(r)}(h)
$$

where

and

$$
\bar{u}^{(r+1)}=\left(u^{(r+1)}\left(x_{1}\right), u^{(r+1)}\left(x_{2}\right), \ldots, u^{(r+1)}\left(x_{N-1}\right)\right)^{T}
$$

$$
T^{(r)}(h)=\left(T_{1}^{(r)}(h), T_{2}^{(r)}(h), \ldots, T_{N-1}^{(r)}(h)\right)^{T} .
$$

Since

$$
A^{(r)} U^{(r+1)}=d^{(r)}
$$

we get

$$
A^{(r)}\left(\bar{u}^{(r+1)}-U^{(r+1)}\right)=T^{(r)}(h)
$$

that is,

$$
A^{(r)} E^{(r+1)}=T^{(r)}(h),
$$

where $E^{(r+1)}=\left(E_{1}^{(r+1)}, E_{2}^{(r+1)}, \ldots, E_{N-1}^{(r+1)}\right), E_{i}^{(r+1)}=u^{(r+1)}\left(x_{i}\right)-U_{i}^{(r+1)}$. Consequently, we obtain that

$$
E^{(r+1)}=A^{(r)^{-1}} T^{(r)}(h) .
$$

From the definition of multiplication of a matrix by its inverse, we have

$$
\sum_{i=1}^{N-1} A^{(r)}{ }_{j, i}^{-1} \mathcal{S}_{i}^{(r)}=1, j=1,2, \ldots, N-1,
$$

where $A_{j, i}^{(r)}{ }_{j, i}^{-1}$ is the $(j, i)$-th element of the matrix $A^{(r)^{-1}}$. Hence, we have

$$
\sum_{i=1}^{N-1} A_{j, i}^{(r)} \leq \frac{1}{\min _{1 \leq i \leq N-1} \mathcal{S}_{i}^{(r)}}=\frac{1}{C_{i_{0}}^{(r)} h^{2}},
$$

where $C_{i_{0}}^{(r)}$ is a constant. We can write

$$
E_{j}^{(r+1)}=\sum_{i=1}^{N-1} A^{(r)}{ }_{j, i}^{-1} T_{i}^{(r)}(h), j=1,2, \ldots, N-1,
$$

and hence $\left|E_{j}^{(r+1)}\right| \leq \frac{K^{(r)} h^{6}}{C_{i_{0}}^{(r)} h^{2}}$, where $K^{(r)}$ is a constant. Therefore, we have

$$
\left\|E^{(r+1)}\right\|_{\infty}=\mathcal{O}\left(h^{4}\right)
$$


which shows that the proposed numerical scheme is of fourth-order convergent for linear BVPs.

\section{Numerical experiments}

For sufficiently small $h$ (such that $B^{(r)}$ is invertible), and by taking $\alpha$ (which makes $A^{(r)}$ invertible and row sums of $A^{(r)}$ positive) that satisfies $|\alpha|<h^{6}$, we can compute the numerical solutions of BVPs.

The numerical solutions of the nonlinear boundary-value problems are computed as follows: For each fixed $N$, by taking the initial approximation $U_{0}^{(0)}, U_{1}^{(0)}, \ldots, U_{N}^{(0)}$, we can compute the numerical solutions $U^{(r+1)}=\left(U_{1}^{(r+1)}, U_{2}^{(r+1)}, \ldots, U_{N-1}^{(r+1)}\right), r=0,1, \ldots$ of the discretized systems (2.11), (2.8), and (2.12).

We take sufficient number of iterations so that the maximum error between the two successive iterations satisfy the following tolerance bound:

$$
\max _{i}\left|U_{i}^{(r+1)}-U_{i}^{(r)}\right|<\text { TOL. }
$$

Once the criterion is satisfied, we consider $U^{(r+1)}$ is the numerical solution $U$ of the nonlinear BVP. To compute the numerical solutions for each of the following nonlinear BVPs, we take TOL $=10^{-15}$. For any fixed-value of $N$, the maximum point-wise error $E^{N}$ will be calculated by

$$
E^{N}=\max _{i}\left|u\left(x_{i}\right)-U_{i}\right|
$$

where $u\left(x_{i}\right)$ and $U_{i}$ are respectively, the exact and numerical solutions of the continuous and discrete problems at $x=x_{i}$. Further, the order of convergence will be calculated by

$$
p^{N}=\log _{2}\left(\frac{E^{N}}{E^{2 N}}\right) \text {. }
$$

From the differential equation given in (1.1), we get the approximation for $u_{x x}\left(x_{i}\right)$ as $M_{i}=f\left(x, U_{i}\right)$. Differentiating (1.1), we get

$$
\begin{aligned}
u_{x x x x}(x)= & -\frac{\partial^{2} f(x, u(x))}{\partial x^{2}}-2 \frac{\partial^{2} f(x, u(x))}{\partial x \partial u} u_{x}(x) \\
& -\frac{\partial^{2} f(x, u(x))}{\partial u^{2}}\left(u_{x}(x)\right)^{2}-\frac{\partial f(x, u(x))}{\partial u} u_{x x}(x)
\end{aligned}
$$

and hence we get approximation of $u_{x x x x}\left(x_{i}\right)$ as

$$
S_{i}=-\frac{\partial^{2} f\left(x_{i}, U_{i}\right)}{\partial x^{2}}-2 \frac{\partial^{2} f\left(x_{i}, U_{i}\right)}{\partial x \partial u} U_{i}^{\prime}-\frac{\partial^{2} f\left(x_{i}, U_{i}\right)}{\partial u^{2}}\left(U_{i}^{\prime}\right)^{2}-\frac{\partial f\left(x_{i}, U_{i}\right)}{\partial u} M_{i},
$$

here $U_{i}^{\prime}$ is the approximation for $u_{x}\left(x_{i}\right)$ and $U_{i}^{\prime}, i=0,1, \ldots, N$ are computed as follows:

$$
\begin{gathered}
U_{i}^{\prime}=\frac{-25 U_{i}+48 U_{i+1}-36 U_{i+2}+16 U_{i+3}-3 U_{i+4}}{12 h}, i=0,1, \\
U_{i}^{\prime}=\frac{U_{i-2}-8 U_{i-1}+8 U_{i+1}-U_{i+2}}{12 h}, i=2,3, \ldots, N-2, \\
U_{i}^{\prime}=\frac{25 U_{i}-48 U_{i-1}+36 U_{i-2}-16 U_{i-3}+3 U_{i-4}}{12 h}, i=N-1, N .
\end{gathered}
$$

We define $E_{2}^{N}$ and $E_{4}^{N}$ as

$$
E_{2}^{N}=\max _{i}\left|u_{x x}\left(x_{i}\right)-M_{i}\right|, \quad E_{4}^{N}=\max _{i}\left|u_{x x x x}\left(x_{i}\right)-S_{i}\right|,
$$

and

$$
p_{2}^{N}=\log _{2}\left(\frac{E_{2}^{N}}{E_{2}^{2 N}}\right), \quad p_{4}^{N}=\log _{2}\left(\frac{E_{4}^{N}}{E_{4}^{2 N}}\right) .
$$


Once we get the values $U_{i}, M_{i}$, and $S_{i}$, we can compute the coefficients given in (2.1). Thus, we get the fractal quintic spline that passes through the solutions of the BVP given in (1.1). From (2.1), we can get the solution of the BVP (1.1) at any point in the interval.

\subsection{Numerical schemes for comparison}

We compare the numerical results of the proposed method with the finite difference method (FDM) and the Numerov's method (NM). We give brief detail about the finite difference method and the Numerov's method in the following subsections.

4.1.1. Finite difference method. Let us consider the BVP given in (2.7). Let $U^{(r+1)}$ be the approximate solution for $u^{(r+1)}(x)$. Substituting

$$
u_{x x}^{(r+1)}(x) \approx \frac{U_{i-1}^{(r+1)}-2 U_{i}^{(r+1)}+U_{i+1}^{(r+1)}}{h^{2}}
$$

in (2.7) and after simplification, we get

$$
U_{i-1}^{(r+1)}+\left[-2+h^{2} q_{i}^{(r)}\right] U_{i}^{(r+1)}+U_{i+1}^{(r+1)}=h^{2} f_{i}^{(r)},
$$

for $i=1,2, \ldots, N-1$, where $q_{i}^{(r)}$ and $f_{i}^{(r)}$ are as given in Section 2.3. Here, $U_{0}^{(r+1)}=\eta_{0}$ and $U_{N}^{(r+1)}=\eta_{1}$.

Now, we can obtain the numerical solution using the condition (4.1).

4.1.2. Numerov's method. The Numerov's method for BVP (1.1) can be written as

$$
-U_{i-1}+2 U_{i}-U_{i+1}=\frac{h^{2}}{12}\left[f_{i-1}+10 f_{i}+f_{i+1}\right]
$$

where $f_{i}=f\left(x_{i}, U_{i}\right), i=0,1, \ldots, N, U_{0}=\eta_{0}$ and $U_{N}=\eta_{1}$. For more details about the Numerov's method, one can see the references $[13,18]$. We can use Newton's method to get the numerical solutions.

Example 4.1. Consider the nonlinear two-point BVP:

$$
\left\{\begin{array}{l}
u_{x x}(x)+\exp (-2 * u(x))=0, \quad x \in(0,1) \\
u(0)=0, \quad u(1)=\log (2)
\end{array}\right.
$$

The exact solution is $u(x)=\log (1+x)$. We calculate the numerical solutions for $N=8,16,32,64,128$. The scaling factors $\alpha=0.9999 h^{6}$ are used to calculate the numerical solutions. The maximum point-wise error $E^{N}$, and the order of convergence $p^{N}, E_{2}^{N}$, $p_{2}^{N}, E_{4}^{N}$, and $p_{4}^{N}$ are given in Table 1 . We have compared the numerical results of the proposed method with the numerical results of finite difference method and Numerov's numerical method. Their results are given in Table 1.

Table 2 represents the solution corresponding to the quintic spline $(\alpha=0)$. Here, we take $U_{0}^{(0)}=0, U_{i}^{(0)}=1, i=1,2, \ldots, N-1, U_{N}^{(0)}=\log (2)$ to compute the numerical solutions.

Figure 1 represents the numerical solution, the corresponding error, and the loglog plot of the error for Example 4.1. From the loglog plot (Figure 1(c)), it can be seen that the 
developed method has fourth-order convergence.

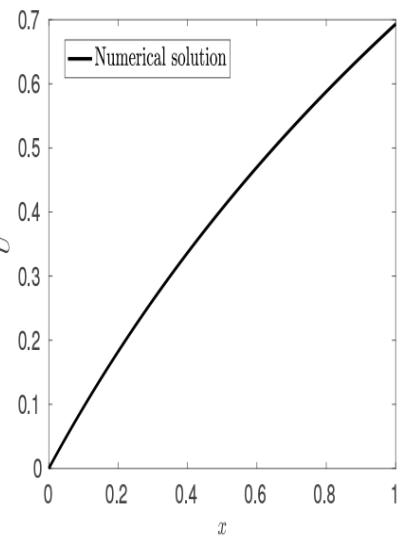

(a) Numerical solution.

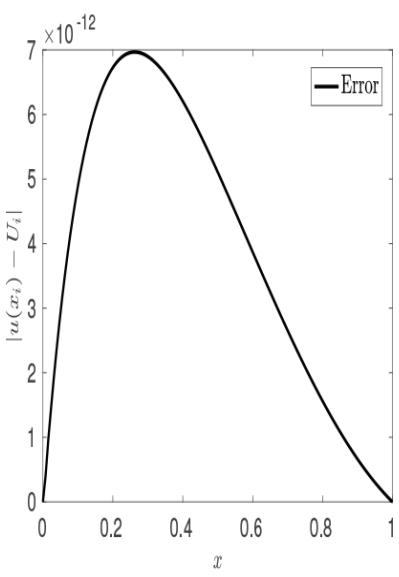

(b) Error.

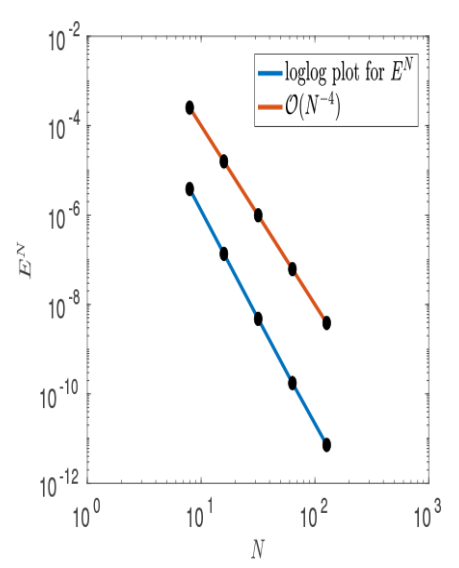

(c) Loglog plot.

Figure 1. Numerical results of Example 4.1 for $N=128$.

Table 1. Maximum point-wise error $E^{N}$, order of convergence $p^{N}, E_{2}^{N}, p_{2}^{N}, E_{4}^{N}$, and $p_{4}^{N}$ corresponding to Example 4.1.

\begin{tabular}{|c|c|c|c|c|c|}
\hline$N$ & 8 & 16 & 32 & 64 & 128 \\
\hline$E^{N}$ & $3.7039 \mathrm{e}-06$ & $1.3093 \mathrm{e}-07$ & $4.6024 \mathrm{e}-09$ & $1.6823 \mathrm{e}-10$ & $6.9676 \mathrm{e}-12$ \\
\hline$p^{N}$ & 4.8222 & 4.8303 & 4.7739 & 4.5936 & \\
\hline$E_{2}^{N}$ & $3.6039 \mathrm{e}-06$ & $1.3560 \mathrm{e}-07$ & $5.1526 \mathrm{e}-09$ & $2.0841 \mathrm{e}-10$ & $9.3744 \mathrm{e}-12$ \\
\hline$p_{2}^{N}$ & 4.7321 & 4.7179 & 4.6278 & 4.4746 & \\
\hline$E_{4}^{N}$ & $3.8032 \mathrm{e}-03$ & $3.5970 \mathrm{e}-04$ & $2.8433 \mathrm{e}-05$ & $2.0142 \mathrm{e}-06$ & $1.3430 \mathrm{e}-07$ \\
\hline$p_{4}^{N}$ & 3.4024 & 3.6611 & 3.8193 & 3.9066 & \\
\hline$(\mathrm{FDM}) E^{N}$ & $2.2281 \mathrm{e}-04$ & $5.6130 \mathrm{e}-05$ & $1.4060 \mathrm{e}-05$ & $3.5166 \mathrm{e}-06$ & $8.7940 \mathrm{e}-07$ \\
\hline$p^{N}$ & 1.9890 & 1.9972 & 1.9993 & 1.9996 & \\
\hline$(\mathrm{NM}) E^{N}$ & $2.0165 \mathrm{e}-06$ & $1.2867 \mathrm{e}-07$ & $8.0669 \mathrm{e}-09$ & $5.0513 \mathrm{e}-10$ & $3.1574 \mathrm{e}-11$ \\
\hline$p^{N}$ & 3.9701 & 3.9955 & 3.9973 & 3.9999 & \\
\hline
\end{tabular}

Table 2. Maximum point-wise error $E^{N}$, order of convergence $p^{N}, E_{2}^{N}, p_{2}^{N}, E_{4}^{N}$, and $p_{4}^{N}$ corresponding to Example 4.1 with quintic spline $(\alpha=0)$.

\begin{tabular}{|c|c|c|c|c|c|}
\hline$N$ & 8 & 16 & 32 & 64 & 128 \\
\hline$E^{N}$ & $6.0415 \mathrm{e}-07$ & $2.9388 \mathrm{e}-08$ & $2.4273 \mathrm{e}-09$ & $1.6374 \mathrm{e}-10$ & $1.0446 \mathrm{e}-11$ \\
\hline$p^{N}$ & 4.3616 & 3.5978 & 3.8898 & 3.9704 & \\
\hline$E_{2}^{N}$ & $9.5470 \mathrm{e}-07$ & $3.2934 \mathrm{e}-08$ & $2.9499 \mathrm{e}-09$ & $2.0374 \mathrm{e}-10$ & $1.3077 \mathrm{e}-11$ \\
\hline$p_{2}^{N}$ & 4.8574 & 3.4809 & 3.8559 & 3.9616 & \\
\hline$E_{4}^{N}$ & $3.6735 \mathrm{e}-03$ & $3.5614 \mathrm{e}-04$ & $2.8356 \mathrm{e}-05$ & $2.0140 \mathrm{e}-06$ & $1.3444 \mathrm{e}-07$ \\
\hline$p_{4}^{N}$ & 3.3666 & 3.6507 & 3.8155 & 3.9051 & \\
\hline
\end{tabular}


Example 4.2. Consider the BVP

$$
\left\{\begin{array}{l}
u_{x x}(x)-\frac{(2-x) \exp (2 u(x))+(1 /(1+x))}{3}=0, \quad x \in(0,1), \\
u(0)=0, \quad u(1)=\log (1 / 2)
\end{array}\right.
$$

The exact solution is $u(x)=\log (1 /(1+x))$. We calculate the numerical solutions for $N=8,16,32,64,128$. The scaling factors $\alpha=0.9999 h^{6}$ are used to calculate the numerical solutions. The maximum point-wise error $E^{N}$, and the order of convergence $p^{N}, E_{2}^{N}$, $p_{2}^{N}, E_{4}^{N}$, and $p_{4}^{N}$ are given in Table 3 . We have compared the numerical results of developed method with the numerical results of finite difference method and the Numerov's method. Their results are given in Table 3. Table 4 represents the solution corresponding to $\alpha=0$, i.e., for the classical quintic spline case. Here, we take $U_{0}^{(0)}=0, U_{i}^{(0)}=1$, $i=1,2, \ldots, N-1, U_{N}^{(0)}=\log (1 / 2)$ to compute the numerical solutions. Figure 2 represents the numerical solution, the corresponding error and the loglog plot of the error for Example 4.2. Figure 2(c) reveals that the developed method has fouth-order convergence.

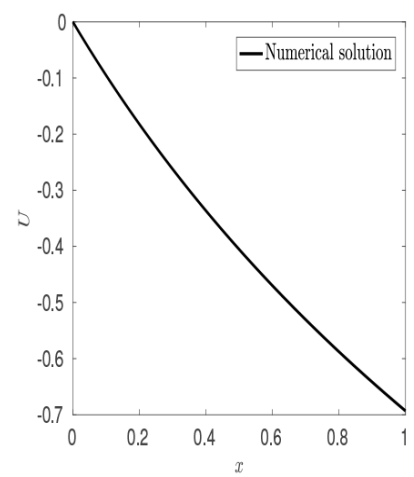

(a) Numerical solution.

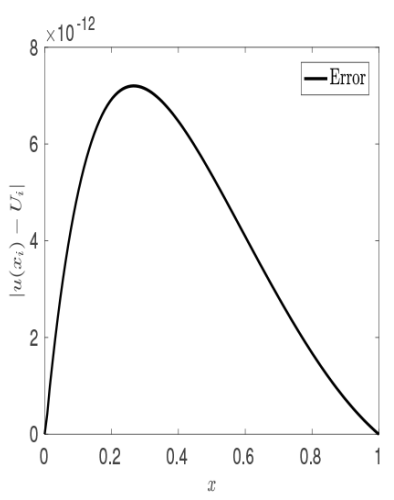

(b) Error.

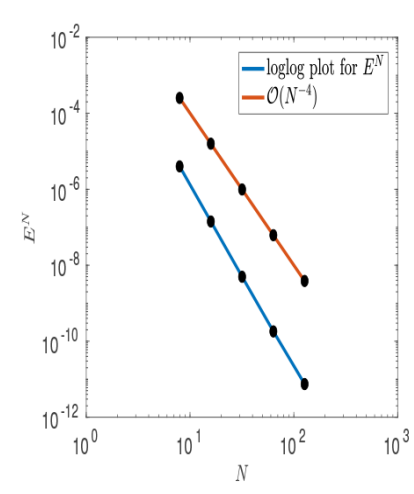

(c) Loglog plot.

Figure 2. Numerical results of Example 4.2 with $N=128$.

Table 3. Maximum point-wise error $E^{N}$, order of convergence $p^{N}, E_{2}^{N}, p_{2}^{N}, E_{4}^{N}$, and $p_{4}^{N}$ corresponding to Example 4.2.

\begin{tabular}{|c|c|c|c|c|c|}
\hline$N$ & 8 & 16 & 32 & 64 & 128 \\
\hline$E^{N}$ & $3.8662 \mathrm{e}-06$ & $1.3680 \mathrm{e}-07$ & $4.8082 \mathrm{e}-09$ & $1.7524 \mathrm{e}-10$ & $7.2015 \mathrm{e}-12$ \\
\hline$p^{N}$ & 4.8207 & 4.8304 & 4.7781 & 4.6048 & \\
\hline$E_{2}^{N}$ & $2.1004 \mathrm{e}-06$ & $8.0168 \mathrm{e}-08$ & $3.1240 \mathrm{e}-09$ & $1.2878 \mathrm{e}-10$ & $5.8584 \mathrm{e}-12$ \\
\hline$p_{2}^{N}$ & 4.7115 & 4.6816 & 4.6004 & 4.4583 & \\
\hline$E_{4}^{N}$ & $3.1728 \mathrm{e}-03$ & $2.9988 \mathrm{e}-04$ & $2.3700 \mathrm{e}-05$ & $1.6787 \mathrm{e}-06$ & $1.1193 \mathrm{e}-07$ \\
\hline$p_{4}^{N}$ & 3.4033 & 3.6615 & 3.8195 & 3.9067 & \\
\hline$(\mathrm{FDM}) E^{N}$ & $2.3261 \mathrm{e}-04$ & $5.8573 \mathrm{e}-05$ & $1.4670 \mathrm{e}-05$ & $3.6702 \mathrm{e}-06$ & $9.1777 \mathrm{e}-07$ \\
\hline$p^{N}$ & 1.9896 & 1.9974 & 1.9989 & 1.9997 & \\
\hline$(\mathrm{NM}) E^{N}$ & $2.1034 \mathrm{e}-06$ & $1.3382 \mathrm{e}-07$ & $8.4017 \mathrm{e}-09$ & $5.2577 \mathrm{e}-10$ & $3.2865 \mathrm{e}-11$ \\
\hline$p^{N}$ & 3.9743 & 3.9935 & 3.9982 & 3.9998 & \\
\hline
\end{tabular}


Table 4. Maximum point-wise error $E^{N}$, order of convergence $p^{N}, E_{2}^{N}, p_{2}^{N}, E_{4}^{N}$, and $p_{4}^{N}$ corresponding to Example 4.2 with quintic spline $(\alpha=0)$.

\begin{tabular}{|c|c|c|c|c|c|}
\hline$N$ & 8 & 16 & 32 & 64 & 128 \\
\hline$E^{N}$ & $6.0740 \mathrm{e}-07$ & $3.0589 \mathrm{e}-08$ & $2.5274 \mathrm{e}-09$ & $1.7045 \mathrm{e}-10$ & $1.0880 \mathrm{e}-11$ \\
\hline$p^{N}$ & 4.3116 & 3.5973 & 3.8903 & 3.9696 & \\
\hline$E_{2}^{N}$ & $5.9990 \mathrm{e}-07$ & $1.9926 \mathrm{e}-08$ & $1.8133 \mathrm{e}-09$ & $1.2596 \mathrm{e}-10$ & $8.1067 \mathrm{e}-12$ \\
\hline$p_{2}^{N}$ & 4.9120 & 3.4579 & 3.8477 & 3.9576 & \\
\hline$E_{4}^{N}$ & $3.0610 \mathrm{e}-03$ & $2.9681 \mathrm{e}-04$ & $2.3633 \mathrm{e}-05$ & $1.6785 \mathrm{e}-06$ & $1.1204 \mathrm{e}-07$ \\
\hline$p_{4}^{N}$ & 3.3664 & 3.6506 & 3.8155 & 3.9051 & \\
\hline
\end{tabular}

Example 4.3. Consider the following nonlinear two-point BVP:

$$
\left\{\begin{array}{l}
u_{x x}(x)-\frac{25 x^{8} \exp (u(x))-20 x^{3}}{4+x^{5}}=0, \quad x \in(0,1), \\
u(0)=-\log (4), \quad u(1)=-\log (5) .
\end{array}\right.
$$

The exact solution is $u(x)=-\log \left(4+x^{5}\right)$. We calculate the numerical solutions for $N=8,16,32,64,128$. The scaling factors $\alpha=0.85 h^{6}$ are used to calculate the numerical solutions. The maximum point-wise error $E^{N}$, order of convergence $p^{N}, E_{2}^{N}, p_{2}^{N}, E_{4}^{N}$, and $p_{4}^{N}$ are given in Table 5. We have compared the numerical results of developed method with the numerical results of finite difference method and the Numerov's method. Their results are given in Table 5. Table 6 represents the solution corresponding to $\alpha=0$. We take $U_{0}^{(0)}=-\log (4), U_{i}^{(0)}=1, i=1,2, \ldots, N-1, U_{N}^{(0)}=-\log (5)$ to compute the numerical solutions. Figure 3 represents the numerical solution, the corresponding error, and the loglog plot of the error for Example 4.3. Figure 3(c) reveals that the developed method has fourth-order convergence.

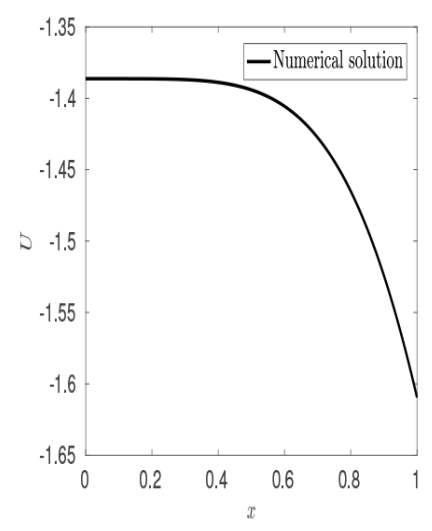

(a) Numerical solution.

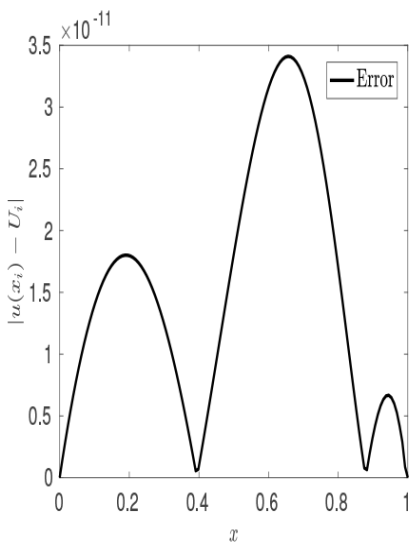

(b) Error.

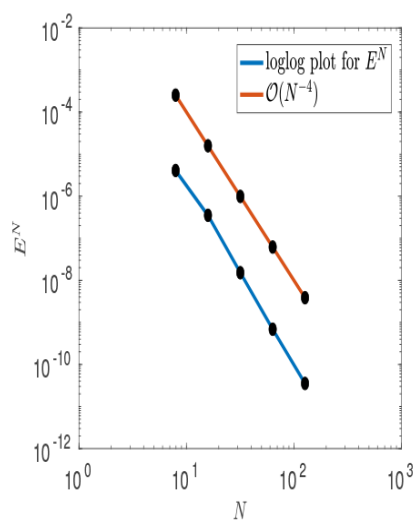

(c) Loglog plot.

Figure 3. Numerical results of Example 4.3 with $N=128$. 
Table 5. Maximum point-wise error $E^{N}$, order of convergence $p^{N}, E_{2}^{N}, p_{2}^{N}, E_{4}^{N}$, and $p_{4}^{N}$ corresponding to Example 4.3.

\begin{tabular}{|c|c|c|c|c|c|}
\hline$N$ & 8 & 16 & 32 & 64 & 128 \\
\hline$E^{N}$ & $3.9439 \mathrm{e}-6$ & $3.3929 \mathrm{e}-07$ & $1.4653 \mathrm{e}-08$ & $6.6424 \mathrm{e}-10$ & $3.4105 \mathrm{e}-11$ \\
\hline$p^{N}$ & 3.5391 & 4.5332 & 4.4633 & 4.2837 & \\
\hline$E_{2}^{N}$ & $4.8434 \mathrm{e}-07$ & $1.0201 \mathrm{e}-07$ & $2.2710 \mathrm{e}-09$ & $8.3241 \mathrm{e}-11$ & $4.8099 \mathrm{e}-12$ \\
\hline$p_{2}^{N}$ & 2.2473 & 5.4893 & 4.7699 & 4.1132 & \\
\hline$E_{4}^{N}$ & $6.8887 \mathrm{e}-02$ & $4.8907 \mathrm{e}-03$ & $2.5980 \mathrm{e}-04$ & $1.4220 \mathrm{e}-05$ & $7.8036 \mathrm{e}-07$ \\
\hline$p_{4}^{N}$ & 3.8161 & 4.2346 & 4.1914 & 4.1876 & \\
\hline$(\mathrm{FDM}) E^{N}$ & $1.1795 \mathrm{e}-03$ & $2.9324 \mathrm{e}-04$ & $7.3024 \mathrm{e}-05$ & $1.8265 \mathrm{e}-05$ & $4.5652 \mathrm{e}-06$ \\
\hline$p^{N}$ & 2.0080 & 2.0056 & 1.9992 & 2.0004 & \\
\hline$(\mathrm{NM}) E^{N}$ & $3.0070 \mathrm{e}-05$ & $1.8480 \mathrm{e}-06$ & $1.1585 \mathrm{e}-07$ & $7.2337 \mathrm{e}-09$ & $4.5198 \mathrm{e}-10$ \\
\hline$p^{N}$ & 4.0242 & 3.9956 & 4.0014 & 4.0004 & \\
\hline
\end{tabular}

Table 6. Maximum point-wise error $E^{N}$, order of convergence $p^{N}, E_{2}^{N}, p_{2}^{N}, E_{4}^{N}$, and $p_{4}^{N}$ corresponding to Example 4.3 with quintic spline $(\alpha=0)$.

\begin{tabular}{|c|c|c|c|c|c|}
\hline$N$ & 8 & 16 & 32 & 64 & 128 \\
\hline$E^{N}$ & $1.0857 \mathrm{e}-05$ & $7.0970 \mathrm{e}-07$ & $4.0662 \mathrm{e}-08$ & $2.4478 \mathrm{e}-09$ & $1.5124 \mathrm{e}-10$ \\
\hline$p^{N}$ & 3.9352 & 4.1255 & 4.0541 & 4.0165 & \\
\hline$E_{2}^{N}$ & $1.7413 \mathrm{e}-06$ & $1.6518 \mathrm{e}-07$ & $7.0721 \mathrm{e}-09$ & $3.9525 \mathrm{e}-10$ & $2.3900 \mathrm{e}-11$ \\
\hline$p_{2}^{N}$ & 3.3981 & 4.5458 & 4.1613 & 4.0477 & \\
\hline$E_{4}^{N}$ & $6.9280 \mathrm{e}-02$ & $4.9109 \mathrm{e}-03$ & $2.6117 \mathrm{e}-04$ & $1.4299 \mathrm{e}-05$ & $7.8595 \mathrm{e}-07$ \\
\hline$p_{4}^{N}$ & 3.8184 & 4.2329 & 4.1910 & 4.1854 & \\
\hline
\end{tabular}

\section{Conclusion}

In this article, with the help of fractal quintic spline a new numerical scheme is proposed for solving nonlinear two-point BVPs. By using the quasilinearization technique, we convert the nonlinear BVP into a sequence of linear BVPs. Then, we obtain the numerical solutions of these linear BVPs using the proposed numerical method. Convergence analysis of the numerical method is derived. The proposed numerical scheme has fourthorder convergence. Numerical examples are presented in supporting the theoretical error estimates.

Acknowledgment. The authors wish to acknowledge the referees for their valuable comments. The first author is grateful to the Ministry of Human Resource Development, India for providing the research fellowship and Indian Institute of Technology Guwahati, India for the support provided during the period of this work.

\section{References}

[1] M. Baccouch, A superconvergent local discontinuous Galerkin method for nonlinear two-point boundary-value problems, Numer. Algor. 79 (3), 697-718, 2018.

[2] M. Baccouch, An adaptive local discontinuous Galerkin method for nonlinear twopoint boundary-value problems, Numer. Algor. 2019, doi:10.1007/s11075-019-00794-8.

[3] M.F. Barnsley, Fractal functions and interpolation, Constr. Approx. 2 (1), 303-329, 1986. 
[4] M.F. Barnsley and A.N. Harrington, The calculus of fractal interpolation functions, J. Approx. Theory 57 (1), 14-34, 1989.

[5] R.E. Bellman and R.E. Kalaba, Quasilinearization and Nonlinear Boundary-Value Problems, American Elsevier, New York, 1965.

[6] R. Bhatia, L. Elsner, and G. Krause, Bounds for the variation of the roots of a polynomial and the eigenvalues of a matrix, Linear Algebra Appl. 142, 195-209, 1990.

[7] S.K. Bhatta and K.S. Sastri, A sixth order spline procedure for a class of nonlinear boundary-value problems, Int. J. Comput. Math. 49 (3-4), 255-271, 1993.

[8] S.K. Bhatta and K.S. Sastri, Symmetric spline procedures for boundary-value problems with mixed boundary conditions, J. Comput. Appl. Math. 45 (3), 237-250, 1993.

[9] A.K.B. Chand and G.P. Kapoor, Generalized cubic spline fractal interpolation functions, SIAM J. Numer. Anal. 44 (2), 655-676, 2006.

[10] A.K.B. Chand and P. Viswanathan, A constructive approach to cubic hermite fractal interpolation function and its constrained aspects, BIT $\mathbf{5 3}$ (4), 841-865, 2013.

[11] M.M. Chawla, A sixth order tridiagonal finite difference method for nonlinear twopoint boundary-value problems, BIT 17 (2), 128-133, 1977.

[12] M.M. Chawla, An eighth order tridiagonal finite difference method for nonlinear twopoint boundary-value problems, BIT 17 (3), 281-285, 1977.

[13] M.M. Chawla and P.N. Shivakumar, Numerov's method for nonlinear two-point boundary-value problems, Int. J. Comput. Math. 17 (2), 167-176, 1985.

[14] M.M. Chawla and R. Subramanian, A new fourth-order cubic spline method for nonlinear two-point boundary-value problems, Int. J. Comput. Math. 22 (3-4), 321-341, 1987.

[15] M.M. Chawla and R. Subramanian, A new fourth-order cubic spline method for second-order nonlinear two-point boundary-value problems, J. Comput. Appl. Math. 23 (1), 1-10, 1988.

[16] M.M. Chawla, R. Subramanian, and P.N. Shivakumar, Numerov's method for nonlinear two-point boundary-value problems II. monotone approximations, Int. J. Comput. Math. 26 (3-4), 219-227, 1989.

[17] U. Erdogan and T. Ozis, A smart nonstandard finite difference scheme for second order nonlinear boundary-value problems, J. Comput. Phys. 230 (17), 6464-6474, 2011.

[18] P. Henrici, Discrete variable methods in ordinary differential equations, John Wiley and Sons, New York, 1962.

[19] M.K. Kadalbajoo and K.C. Patidar, Spline techniques for solving singularly-perturbed nonlinear problems on nonuniform grids, J. Optim. Theory Appl. 114 (3), 573-591, 2002.

[20] M. Lees, Discrete method for nonlinear two-point boundary-value problems, in: Numerical Solution of Partial Differential Equations, ed. J.H. Bramble, Academic Press, New York, 1966.

[21] L.B. Liu, H.W. Liu, and Y. Chen, Polynomial spline approach for solving second-order boundary-value problems with Neumann conditions, Appl. Math. Comput. 217 (16), 6872-6882, 2011.

[22] J. Rashidinia, R. Mohammadi, and R. Jalilian, Spline solution of nonlinear singular boundary-value problems, Int. J. Comput. Math. 85 (1), 39-52, 2008.

[23] A.S.V. Ravikanth and V. Bhattacharya, Cubic spline for a class of nonlinear singular boundary-value problems arising in physiology, Appl. Math. Comput. 174 (1), 768$774,2006$.

[24] S.B.G. Karakoç, N.M. Yagmurlu, and Y. Ucar, Numerical approximation to a solution of the modified regularized long wave equation using quintic B-splines, Bound. Value Probl. 2013 (1), 2013. 
[25] I.A. Tirmizi and E.H. Twizell, Higher-order finite-difference methods for nonlinear second-order two-point boundary-value problems, Appl. Math. Lett. 15 (7), 897-902, 2002.

[26] H. Zeybek and S.B.G. Karakoç, Application of the collocation method with B-splines to the GEW equation, Electron. Trans. Numer. Anal. 46, 71-88, 2017. 